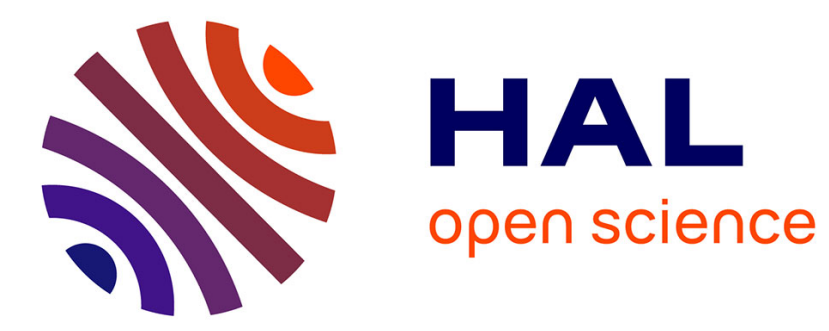

\title{
Operational approach for biharmonic equations in $\mathrm{Lp}$ -spaces
}

Alexandre Thorel

\section{To cite this version:}

Alexandre Thorel. Operational approach for biharmonic equations in Lp -spaces. Journal of Evolution Equations, In press, 20, pp. 631-657. 10.1007/s00028-019-00536-2 . hal-02170037v2

\section{HAL Id: hal-02170037 \\ https://hal.science/hal-02170037v2}

Submitted on 1 Oct 2019

HAL is a multi-disciplinary open access archive for the deposit and dissemination of scientific research documents, whether they are published or not. The documents may come from teaching and research institutions in France or abroad, or from public or private research centers.
L'archive ouverte pluridisciplinaire HAL, est destinée au dépôt et à la diffusion de documents scientifiques de niveau recherche, publiés ou non, émanant des établissements d'enseignement et de recherche français ou étrangers, des laboratoires publics ou privés. 


\title{
Operational approach for biharmonic equations in $L^{p}$-spaces
}

\author{
Alexandre Thorel \\ Normandie Univ, UNIHAVRE, LMAH, FR-CNRS-3335, ISCN, 76600 Le Havre, France. \\ alexandre.thorel@univ-lehavre.fr
}

\begin{abstract}
In this work, we study the existence, uniqueness and maximal $L^{p}$-regularity of the solution of different biharmonic problems. We rewrite these problems by a fourth order operational equation and different boundary conditions, set in a cylindrical $n$-dimensional spatial region $\Omega$ of $\mathbb{R}^{n}$. To this end, we give an explicit representation formula, using analytic semigroups, and invert explicitly a determinant operator in $L^{p}$-spaces thanks to $\mathcal{E}_{\infty}$ functional calculus and operator sums theory.
\end{abstract}

Key Words and Phrases: Operational differential equations, functional calculus, analytic semigroups, interpolation spaces, maximal regularity.

2010 Mathematics Subject Classification: 35B65, 35C15, 35J40, 35R20, 47A60, 47D06.

\section{Introduction}

In this article we develop an operator approach to study some properties of the solutions of the biharmonic problem

$$
\Delta^{2} u=f,
$$

set on a straight cylinder $\Omega=(a, b) \times \omega$ where $\omega \subset \mathbb{R}^{n-1}$ denotes a bounded domain with a $C^{2}$ boundary and $n \geq 2$ is a given integer while the function $f \in L^{p}(\Omega)$ with $p \in(1, \infty)$.

Here we are concerned with the existence, uniqueness and $L^{p}$-maximal regularity of the solutions of (1) when this problem is supplemented with various types of non-homogeneous boundary conditions (see below).

Biharmonic problems arise in various applicative fields, including physics, engineering and biology. In [5], [17], or [31], biharmonic equations are used to model elasticity problem where displacement, stress, and strain satisfy (1). One may also refer to [3], [14] or [23] for biharmonic models in electrostatic, to [8], [11] or [32] for applications in plate theory where various different boundary conditions arise. We also refer to [4], [19], [20] or [26] for models in population dynamics.

Coming back to (1), we now specific the boundary conditions that will be studied along this work. First we assume the homogeneous Dirichlet boundary conditions on the lateral boundary of $\Omega$, namely $(a, b) \times \partial \omega$. This reads as

$$
u(x, \zeta)=\Delta u(x, \zeta)=0, \quad(x, \zeta) \in(a, b) \times \partial \omega .
$$

As far as the boundary conditions on $\{a, b\} \times \omega$, we shall study the following five different types

$$
\left\{\begin{aligned}
u(a, y) & =\varphi_{1}(y), \quad u(b, y) & =\varphi_{2}(y), \quad y \in \omega, \\
\partial_{x x} u(a, y) & =\varphi_{3}(y), \quad \partial_{x x} u(b, y) & =\varphi_{4}(y), \quad y \in \omega,
\end{aligned}\right.
$$

or

$$
\left\{\begin{aligned}
\partial_{x} u(a, y) & =\varphi_{1}(y), \quad \partial_{x} u(b, y)=\varphi_{2}(y), \quad y \in \omega \\
\Delta u(a, y) & =\varphi_{3}(y), \quad \Delta u(b, y)=\varphi_{4}(y), \quad y \in \omega
\end{aligned}\right.
$$

or

$$
\left\{\begin{aligned}
u(a, y) & =\varphi_{1}(y), \quad u(b, y)=\varphi_{2}(y), \quad y \in \omega, \\
\partial_{x} u(a, y) & =\varphi_{3}(y), \quad \partial_{x} u(b, y)=\varphi_{4}(y), \quad y \in \omega,
\end{aligned}\right.
$$




$$
\left\{\begin{aligned}
\partial_{x} u(a, y) & =\varphi_{1}(y), \quad \partial_{x} u(b, y)=\varphi_{2}(y), \quad y \in \omega \\
\partial_{x x} u(a, y) & =\varphi_{3}(y), \quad \partial_{x x} u(b, y)=\varphi_{4}(y), \quad y \in \omega
\end{aligned}\right.
$$

or

$$
\left\{\begin{aligned}
u(a, y) & =\varphi_{1}(y), \quad u(b, y)=\varphi_{2}(y), \quad y \in \omega \\
\Delta u(a, y) & =\varphi_{3}(y), \quad \Delta u(b, y)=\varphi_{4}(y), \quad y \in \omega .
\end{aligned}\right.
$$

In the above boundary conditions, the functions $\varphi_{1}, \varphi_{2}, \varphi_{3}$ and $\varphi_{4}$ are assumed to belong to $L^{p}(\omega)$

As mentioned above, in this article we develop an operator theoric approach to handle the biharmonic equation (1)-(2) and supplemented with one of the previous boundary conditions. This work can be viewed as a continuation of the analysis proposed in [20] where the equation

$$
k_{2} \Delta^{2} u-k_{1} \Delta u=f,
$$

has been studied using a suitable operational framework. Note that in the aforementioned work, the condition $k_{1} \neq 0$ plays a crucial role in this analysis. So that the present work extends this previous analysis by taking into account this limit case $k_{1}=0$.

Here, to handle (1)-(2) and one of the previous boundary conditions, we reformulate this problem as a suitable fouth order abstract differential equation that allow us to derive existence, uniquess and also to provide necessary and sufficient conditions on the boundary functions $\varphi_{i}$ arising in the previous boundary conditions, ensuring maximal $L^{p}$-regularity.

More specifically let us define $A_{0}$, the Laplace operator in $\mathbb{R}^{n-1}, n \in \mathbb{N} \backslash\{0,1\}$, on the Banach space $L^{q}(\omega), q \in(1,+\infty)$, with homogeneous Dirichlet boundary conditions on $\partial \omega$. Then, $A_{0}$ reads as:

$$
\left\{\begin{array}{l}
D\left(A_{0}\right):=\left\{\psi \in W^{2, p}(\omega): \psi=0 \text { on } \partial \omega\right\} \\
\forall \psi \in D\left(A_{0}\right), \quad A_{0} \psi=\Delta_{y} \psi
\end{array}\right.
$$

Thus, equation (1) could be reformulated as the fourth order operational differential equation,

$$
u^{(4)}(x)+2 A_{0} u^{\prime \prime}(x)+A_{0}^{2} u(x)=f(x), \quad \text { for a.e. } \quad x \in(a, b),
$$

where $f \in L^{p}\left(a, b ; L^{q}(\omega)\right), p \in(1,+\infty)$, with $u(x):=u(x, \cdot)$ and $f(x):=f(x, \cdot)$. Then, we consider the generalization of this equation with $(A, D(A))$, instead of $\left(A_{0}, D\left(A_{0}\right)\right)$ :

$$
u^{(4)}(x)+2 A u^{\prime \prime}(x)+A^{2} u(x)=f(x), \quad \text { for a. e. } \quad x \in(a, b),
$$

where "- $A$ " is a BIP operator of angle $\theta_{A} \in(0, \pi)$ on a UMD space $X$, see Section 2 below for the definitions of BIP operator and UMD spaces, and $f \in L^{p}(a, b ; X), p \in(1,+\infty)$.

More precisely, we will study equation (4) under the following boundary conditions:

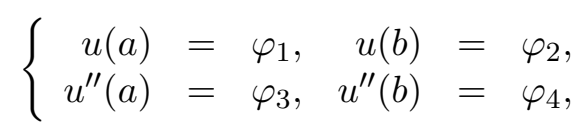

or

$$
\left\{\begin{aligned}
u^{\prime}(a) & =\varphi_{1}, & u^{\prime}(b) & =\varphi_{2} \\
u^{\prime \prime}(a)+A u(a) & =\varphi_{3}, & u^{\prime \prime}(b)+A u(b) & =\varphi_{4}
\end{aligned}\right.
$$

or

$$
\left\{\begin{aligned}
u(a) & =\varphi_{1}, \quad u(b)=\varphi_{2} \\
u^{\prime}(a) & =\varphi_{3}, \quad u^{\prime}(b)=\varphi_{4}
\end{aligned}\right.
$$

or

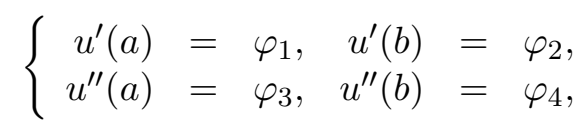

or

$$
\left\{\begin{aligned}
u(a) & =\varphi_{1}, & u(b) & =\varphi_{2} \\
u^{\prime \prime}(a)+A u(a) & =\varphi_{3}, & u^{\prime \prime}(b)+A u(b) & =\varphi_{4}
\end{aligned}\right.
$$


where $\varphi_{1}, \varphi_{2}, \varphi_{3}, \varphi_{4} \in X$.

Recall that $u$ is a classical solution of equation (4) if $u$ is a solution of (4) satisfying

$$
u \in W^{4, p}(a, b ; X) \cap L^{p}\left(a, b ; D\left(A^{2}\right)\right) \quad \text { and } \quad u^{\prime \prime} \in L^{p}(a, b ; D(A)),
$$

Moreover, for $i \in\{1,2,3,4,5\}$, we say that $u$ is a classical solution of problem (4)-(BCi), if $u$ is a classical solution of (4) satisfying (BCi).

Our main result is given by Theorem 2.8 in which we give necessary and sufficient conditions for the existence and uniqueness of a classical solution $u$ of problem (4)-(BCi).

As a byproduct of our analysis, we also obtain an explicit representation formula of the solutions of (4) and also a regularity result of the terms composed by a polynomial function and an analytic semigroup.

The paper is organised as follows:

In Section 2, we detail our hypotheses on a UMD Banach space $X$ and on the operator $A$. Then, we give our main results. As a consequence, we obtain Corollary 2.9 which establishes the existence and the uniqueness of the solution $u$ in $W^{4, p}(\Omega)$ of problem (1)-(2)-(3.3).

In Section 3, we recall some well-known results useful for the next sections.

In Section 4, we establish some preliminary results. First of all, in Section 4.1, we make explicit, in Proposition 4.1, a general representation formula for classical solutions. In Section 4.2, we give a very useful technical result in Proposition 4.2 concerning the regularity of the product between a polynomial function and an analytic semigroup. In Section 4.3, we give some traces result in Proposition 4.3. Then, in Section 4.4, we use functional calculus to prove an invertibility result in Proposition 4.5 which is very useful for the last section.

Finally, in the last section, we prove our main result, Theorem 2.8 , which completes Theorem 2.6 by showing the existence and the uniqueness of the classical solution $u$ of problems (4)-(BC2), (4)-(BC3) and (4)-(BC4).

\section{Assumptions and statement of results}

\section{$2.1 \quad$ Hypotheses}

Throughout the article, $(X,\|\cdot\|)$ is a complex Banach space. We first give some useful definitions before stating our hypotheses.

Definition 2.1. A Banach space $X$ is a UMD space if and only if for all $p \in(1,+\infty)$, the Hilbert transform is bounded from $L^{p}(\mathbb{R}, X)$ into itself (see [1] and [2]).

Definition 2.2. A closed linear operator $T_{1}$ in $X$ is called sectorial of angle $\theta \in[0, \pi)$ if

$$
\begin{aligned}
\text { i) } & \sigma\left(T_{1}\right) \subset \overline{S_{\theta}}, \\
\text { ii) } & \forall \theta^{\prime} \in(\theta, \pi), \quad \sup \left\{\left\|\lambda\left(\lambda I-T_{1}\right)^{-1}\right\|_{\mathcal{L}(X)}: \lambda \in \mathbb{C} \backslash \overline{S_{\theta^{\prime}}}\right\}<+\infty,
\end{aligned}
$$

where

$$
S_{\theta}:= \begin{cases}\{z \in \mathbb{C}: z \neq 0 \text { and }|\arg (z)|<\theta\} & \text { if } \theta \in(0, \pi) \\ (0,+\infty) & \text { if } \theta=0\end{cases}
$$

see [15], p. 19.

Definition 2.3. We denote by $\operatorname{BIP}(X, \theta), \theta \in[0, \pi)$, see [28], p. 431, the class of sectorial injective operators $T_{1}$ in $X$ such that

i) $\overline{D\left(T_{1}\right)}=\overline{R\left(T_{1}\right)}=X$,

ii) $\forall s \in \mathbb{R}, \quad T_{1}^{i s} \in \mathcal{L}(X)$,

iii) $\exists C \geq 1, \forall s \in \mathbb{R}, \quad\left\|T_{1}^{i s}\right\|_{\mathcal{L}(X)} \leq C e^{|s| \theta}$. 
We are now in position to describe our assumptions:

$$
\begin{aligned}
& \left(H_{1}\right) \quad X \text { is a UMD space, } \\
& \left(H_{2}\right) \quad A \text { is a closed linear operator in } X \text { and } 0 \in \rho(A), \\
& \left(H_{3}\right) \quad-A \in \operatorname{BIP}\left(X, \theta_{A}\right) \text { for some } \theta_{A} \in(0, \pi),
\end{aligned}
$$

\section{Remark 2.4.}

1. The operator $A_{0}$, given by (3), satisfies all the previous hypotheses with $X=L^{q}(\omega), q \in(1,+\infty)$. From [30], Proposition 3, p. 207, $X$ satisfies $\left(H_{1}\right)$ and from [12], Theorem 9.15, p. 241 and Lemma 9.17, p.242, $A_{0}$ satisfies $\left(H_{2}\right)$. Moreover, $\left(H_{3}\right)$ is satisfied for every $\theta_{A} \in(0, \pi)$, from [29], Theorem C, p. 166-167.

2. If we consider the real case (with $-A>0$ ), then we can solve equation (1) with the help of the roots $\pm \sqrt{-A}$ of the characteristic equation

$$
x^{4}+2 A x^{2}+A^{2}=0,
$$

this is why, in our operational case, we consider the operator

$$
M:=-\sqrt{-A} .
$$

From $\left(H_{3}\right)$, since $-A$ is a sectorial operator, then the existence of $M$ is ensured, see [15], p. 61 .

3. Due to $\left(H_{2}\right)$ we deduce that $0 \in \rho(M)$, from [15], Proposition 3.1.1, p. 62 .

4. From [15], Proposition 3.2.1, p. 71 and $\left(H_{3}\right)$, we deduce that

$$
-M \in \operatorname{BIP}\left(X, \theta_{A} / 2\right) \text {. }
$$

\subsection{The main results}

We first need some definitions and results concerning real interpolation spaces, the definition of which is given in [6], [13], [24] or in [33]. We consider here the following particular case:

Definition 2.5. Let $T_{2}: D\left(T_{2}\right) \subset X \longrightarrow X$ be a linear operator such that

$$
(0,+\infty) \subset \rho\left(T_{2}\right) \quad \text { and } \quad \exists C>0: \forall t>0, \quad\left\|t\left(T_{2}-t I\right)^{-1}\right\|_{\mathcal{L}(X)} \leqslant C .
$$

Let $k \in \mathbb{N} \backslash\{0\}, \theta \in(0,1)$ and $q \in[1,+\infty]$. We will use the real interpolation spaces

$$
\left(D\left(T_{2}^{k}\right), X\right)_{\theta, q}=\left(X, D\left(T_{2}^{k}\right)\right)_{1-\theta, q},
$$

defined, for instance, in [24], or in [25].

In particular, for $k=1$, we have the following characterization

$$
\left(D\left(T_{2}\right), X\right)_{\theta, q}:=\left\{\psi \in X: t \longmapsto t^{1-\theta}\left\|T_{2}\left(T_{2}-t I\right)^{-1} \psi\right\|_{X} \in L_{*}^{q}(0,+\infty)\right\},
$$

where $L_{*}^{q}(0,+\infty)$ is given by

$$
L_{*}^{q}(0,+\infty):=\left\{f \in L^{q}(0,+\infty):\left(\int_{0}^{+\infty}\|f(t)\|^{q} \frac{d t}{t}\right)^{1 / q}<+\infty\right\}, \quad \text { for } q \in[1,+\infty),
$$

and for $q=+\infty$, by

$$
L_{*}^{\infty}(0,+\infty):=\sup _{t \in(0,+\infty)}\|f(t)\|,
$$

see [6] p. 325, or [13], p. 665, Teorema 3, or section 1.14 of [33], where this space is denoted by $\left(X, D\left(T_{2}\right)\right)_{1-\theta, q}$. Note that we can also characterize the space $\left(D\left(T_{2}\right), X\right)_{\theta, q}$ taking into account the Osservazione, p. 666, in [13].

We set also, for any $k \in \mathbb{N} \backslash\{0\}$

$$
\left(X, D\left(T_{2}\right)\right)_{k+\theta, q}:=\left\{\psi \in D\left(T_{2}^{k}\right): T_{2}^{k} \psi \in\left(X, D\left(T_{2}\right)\right)_{\theta, q}\right\} .
$$


We recall the following Theorem obtained in [20] (this result is given for $k \neq 0$ but clearly remains true if $k=0)$.

Theorem 2.6 (see [20]). Let $f \in L^{p}(a, b ; X)$ with $a, b \in \mathbb{R}, a<b$ and $p \in(1,+\infty)$. Assume that $\left(H_{1}\right),\left(H_{2}\right)$ and $\left(H_{3}\right)$ hold. Then

1. there exists a unique classical solution $u$ of (4)-(BC1) if and only if

$$
\varphi_{1}, \varphi_{2} \in D(A) \quad \text { and } \quad A \varphi_{1}, A \varphi_{2}, \varphi_{3}, \varphi_{4} \in(D(A), X)_{\frac{1}{2 p}, p} .
$$

This unique classical solution is called $F_{\Phi, f}$ with $\Phi:=\left(\varphi_{1}, \varphi_{2}, \varphi_{3}, \varphi_{4}\right)$ and is explicitly described by

$$
\begin{aligned}
F_{\Phi, f}(x):= & e^{(x-a) M} Z \varphi_{1}+e^{(b-x) M} Z \varphi_{2} \\
& -\frac{1}{2} e^{(x-a) M} Z M^{-1} \int_{a}^{b} e^{(s-a) M} v_{0}(s) d s \\
& -\frac{1}{2} e^{(b-x) M} Z M^{-1} \int_{a}^{b} e^{(b-s) M} v_{0}(s) d s \\
& +\frac{1}{2} M^{-1} \int_{a}^{x} e^{(x-s) M} v_{0}(s) d s+\frac{1}{2} M^{-1} \int_{x}^{b} e^{(s-x) M} v_{0}(s) d s \\
& -e^{(b-x) M} e^{(b-a) M} Z \varphi_{1}-e^{(x-a) M} e^{(b-a) M} Z \varphi_{2} \\
& +\frac{1}{2} e^{(x-a) M} Z e^{(b-a) M} M^{-1} \int_{a}^{b} e^{(b-s) M} v_{0}(s) d s \\
& +\frac{1}{2} e^{(b-x) M} Z e^{(b-a) M} M^{-1} \int_{a}^{b} e^{(s-a) M} v_{0}(s) d s, \quad x \in[a, b],
\end{aligned}
$$

where

$$
\begin{aligned}
v_{0}(x):= & e^{(x-a) M} Z\left(\varphi_{3}+A \varphi_{1}\right)+e^{(b-x) M} Z\left(\varphi_{4}+A \varphi_{2}\right) \\
& -\frac{1}{2} e^{(x-a) M} Z M^{-1} \int_{a}^{b} e^{(s-a) M} f(s) d s-\frac{1}{2} e^{(b-x) M} Z M^{-1} \int_{a}^{b} e^{(b-s) M} f(s) d s \\
& +\frac{1}{2} M^{-1} \int_{a}^{x} e^{(x-s) M} f(s) d s+\frac{1}{2} M^{-1} \int_{x}^{b} e^{(s-x) M} f(s) d s \\
& -e^{(b-x) M} e^{(b-a) M} Z\left(\varphi_{3}+A \varphi_{1}\right)-e^{(x-a) M} e^{(b-a) M} Z\left(\varphi_{4}+A \varphi_{2}\right) \\
& +\frac{1}{2} e^{(x-a) M} Z e^{(b-a) M} M^{-1} \int_{a}^{b} e^{(b-s) M} f(s) d s \\
& +\frac{1}{2} e^{(b-x) M} Z e^{(b-a) M} M^{-1} \int_{a}^{b} e^{(s-a) M} f(s) d s, \quad x \in[a, b],
\end{aligned}
$$

and $Z:=\left(I-e^{2(b-a) M}\right)^{-1}$.

The existence of $Z$ is ensured from [25], Proposition 2.3.6, p. 60.

2. there exists a unique classical solution $u$ of (4)-(BC5) if and only if

$$
\varphi_{1}, \varphi_{2} \in D(A) \quad \text { and } \quad A \varphi_{1}, A \varphi_{2}, \varphi_{3}, \varphi_{4} \in(D(A), X)_{\frac{1}{2 p}, p} .
$$

In this case the unique solution is $u=F_{\left(\varphi_{1}, \varphi_{2}, \varphi_{3}-A \varphi_{1}, \varphi_{4}-A \varphi_{2}\right), f}$.

\section{Remark 2.7.}

1. The previous results use the works of [6] and [7]. 
2. In the previous Theorem, statement 2. is obtained from statement 1 . in the following manner: condition (BC5) could be written as follows

$$
\left\{\begin{array}{rlrl}
u(a) & =\varphi_{1}, & u(b) & =\varphi_{2}, \\
u^{\prime \prime}(a) & =\varphi_{3}-A \varphi_{1}, \quad u^{\prime \prime}(b) & =\varphi_{4}-A \varphi_{2} .
\end{array}\right.
$$

From statement 1., there exists a unique classical solution $u$ of problem (4)-(BC5) if and only if

$$
\varphi_{1}, \varphi_{2} \in D(A) \quad \text { and } \quad A \varphi_{1}, A \varphi_{2}, \varphi_{3}-A \varphi_{1}, \varphi_{4}-A \varphi_{2} \in(D(A), X)_{\frac{1}{2 p}, p},
$$

which is equivalent to (11). Thus, we deduce that $u=F_{\left(\varphi_{1}, \varphi_{2}, \varphi_{3}-A \varphi_{1}, \varphi_{4}-A \varphi_{2}\right), f}$.

Now, we give our main results.

Theorem 2.8. Let $f \in L^{p}(a, b ; X)$ with $a<b, a, b \in \mathbb{R}$ and $p \in(1,+\infty)$. Assume that $\left(H_{1}\right),\left(H_{2}\right)$ and $\left(H_{3}\right)$ hold. Then

1. there exists a unique classical solution $u$ of (4)-(BC2) if and only if

$$
\varphi_{1}, \varphi_{2} \in D(A), \quad A \varphi_{1}, A \varphi_{2} \in(D(A), X)_{\frac{1}{2}+\frac{1}{2 p}, p} \quad \text { and } \quad \varphi_{3}, \varphi_{4} \in(D(A), X)_{\frac{1}{2 p}, p} .
$$

Moreover, if, in $\left(H_{3}\right), \theta_{A}<\pi / 2$, then

2. there exists a unique classical solution $u$ of (4)-(BC3) if and only if

$$
\varphi_{1}, \varphi_{2}, \varphi_{3}, \varphi_{4} \in D(A), \quad A \varphi_{1}, A \varphi_{2} \in(D(A), X)_{\frac{1}{2 p}, p} \quad \text { and } \quad A \varphi_{3}, A \varphi_{4} \in(D(A), X)_{\frac{1}{2}+\frac{1}{2 p}, p},
$$

3. there exists a unique classical solution $u$ of (4)-(BC4) if and only if

$$
\varphi_{1}, \varphi_{2} \in D(A), \quad A \varphi_{1}, A \varphi_{2} \in(D(A), X)_{\frac{1}{2}+\frac{1}{2 p}, p} \quad \text { and } \quad \varphi_{3}, \varphi_{4} \in(D(A), X)_{\frac{1}{2 p}, p} .
$$

As a consequence of Theorem 2.8, we can make explicit our problems (4)-(BCi) with $A=A_{0}$ and, for instance, we do it for (4)-(BC3) and solve problem (1)-(2)-(3.3):

Corollary 2.9. Consider a cylindrical domain $\Omega=(a, b) \times \omega$ of $\mathbb{R}^{n}$, where $n \geqslant 2, a, b \in \mathbb{R}, a<b$ and $\omega$ is a bounded open set of $\mathbb{R}^{n-1}$, where $n \in \mathbb{N} \backslash\{0,1\}$, with $C^{2}$ boundary. Let $f \in L^{p}(\Omega)$ with $p \in(1,+\infty)$ and $p>n$. Then, there exists a unique solution $u \in W^{4, p}(\Omega)$ of

$$
\left\{\begin{aligned}
\Delta^{2} u(x, y) & =f(x, y), & & (x, y) \in \Omega \\
u(x, \zeta)=\Delta u(x, \zeta) & =0, & & (x, \zeta) \in(a, b) \times \partial \omega \\
u(a, y) & =\varphi_{1}(y), & & y \in \omega \\
u(b, y) & =\varphi_{2}(y), & & y \in \omega \\
\partial_{x} u(a, y) & =\varphi_{3}(y), & & y \in \omega \\
\partial_{x} u(b, y) & =\varphi_{4}(y), & & y \in \omega,
\end{aligned}\right.
$$

if and only if

$$
\left\{\begin{array}{c}
\varphi_{1}, \varphi_{2}, \varphi_{3}, \varphi_{4} \in W^{2, p}(\omega) \cap W_{0}^{1, p}(\omega) \\
\Delta \varphi_{1}, \Delta \varphi_{2} \in W^{2-\frac{1}{p}, p}(\omega) \cap W_{0}^{1, p}(\omega) \\
\Delta \varphi_{3}, \Delta \varphi_{4} \in W^{1-\frac{1}{p}, p}(\omega) \cap W_{0}^{1, p}(\omega) .
\end{array}\right.
$$

Proof. The proof is quite similar to the one of Corollary 2.7 in [20], see also Corollary 3.6 in [19].

Taking into account the result of Theorem 2.8, we can also obtain anisotropic result by considering $f \in L^{p}\left(a, b ; L^{q}(\omega)\right)$ with $p, q \in(1,+\infty)$. 


\section{Prerequisites}

In this section, we recall some well-known facts, useful in our proofs.

Lemma 3.1 ([13]). Let $T_{2}$ be a linear operator satisfying (6). Let u such that

$$
u \in W^{n, p}\left(a_{1}, b_{1} ; X\right) \cap L^{p}\left(a_{1}, b_{1} ; D\left(T_{2}^{k}\right)\right),
$$

where $a_{1}, b_{1} \in \mathbb{R}$ with $a_{1}<b_{1}, n, k \in \mathbb{N} \backslash\{0\}$ and $p \in(1,+\infty)$. Then for any $j \in \mathbb{N}$ satisfying the Poulsen condition $0<\frac{1}{p}+j<n$ and $s \in\left\{a_{1}, b_{1}\right\}$, we have

$$
u^{(j)}(s) \in\left(D\left(T_{2}^{k}\right), X\right)_{\frac{j}{n}+\frac{1}{n p}, p} .
$$

This result is proved in [13], Theorem 2'.

Lemma 3.2. [33] Let $\psi \in X$ and $T_{2}$ be a generator of a bounded analytic semigroup in $X$ with $0 \in \rho\left(T_{2}\right)$. Then, for any $n \in \mathbb{N} \backslash\{0\}$ and $p \in[1,+\infty]$, the next properties are equivalent:

1. $x \mapsto e^{(x-a) T_{2}} \psi \in W^{n, p}(a, b ; X)$

2. $x \mapsto T_{2}^{n} e^{(x-a) T_{2}} \psi \in L^{p}(a, b ; X)$

3. $x \mapsto e^{(x-a) T_{2}} \psi \in W^{n, p}(a,+\infty ; X)$

4. $x \mapsto T_{2}^{n} e^{(x-a) T_{2}} \psi \in L^{p}(a,+\infty ; X)$

5. $\psi \in D\left(T_{2}^{n-1}\right) \quad$ and $\quad T_{2}^{n-1} \psi \in\left(D\left(T_{2}\right), X\right)_{\frac{1}{p}, p}$

Obviously, statement 1 . is equivalent to statement 2 . and also statement 3 . is equivalent to statement 4. Furthermore, 4. is equivalent to 5., see [33], Theorem, p. 96. Finally, since $T_{2}$ is a generator of a bounded analytic semigroup with $0 \in \rho\left(T_{2}\right)$, we have

$$
\omega_{T_{2}}:=\sup \left\{\operatorname{Re}(\lambda): \lambda \in \sigma\left(T_{2}\right)\right\}<0,
$$

so from Proposition 2.3.1. in [25], p. 56, we have

$$
\exists M \geqslant 1, \exists \delta>0, \forall x \geqslant b, \quad\left\|T_{2}^{n} e^{(x-a) T_{2}}\right\| \leqslant \frac{M}{(x-a)^{n}} e^{-\delta(x-a)},
$$

from which we deduce that statement 2 . is equivalent to statement 4 .

Lemma 3.3 ([20]). Let $V \in \mathcal{L}(X)$ such that $0 \in \rho(I+V)$. Then, there exists $W \in \mathcal{L}(X)$ such that

$$
(I+V)^{-1}=I-W
$$

and $W(X) \subset V(X)$. Moreover, if $T$ is a linear operator in $X$ such that $V(X) \subset D(T)$ and for $\psi \in D(T), T V \psi=V T \psi$, then

$$
\forall \psi \in D(T), \quad W T \psi=T W \psi .
$$

This result is proved in [20], Lemma 5.1.

\section{Preliminary results}

We first note that $F_{0, f}$ defined in Theorem 2.6 is a particular solution of equation (4), this will be used, in the following subsection, to build a general representation formula of the solution of equation (4). Then, in the next subsection, we give a result which allow us to study the regularity of this representation formula. In the third subsection, we give a result on traces of classical solution of (4). Finally, in the last subsection, we give some invertibility results using functional calculus. 


\subsection{Representation formula}

Theorem 2.6 furnishes results of existence and uniqueness of a classical solution of problem (4)-(BC1) and (4)-(BC5). With other boundary conditions, the equation (4) is not easy to study: the first step is to state a representation formula of the solution of equation (4). A similar representation formula has been given in [8] without details, this formula gives a formal solution of equation (4), nevertheless, it has not been proved that this formula leads to formal solution of all the classical solutions of equation (4).

Proposition 4.1. Under $\left(H_{1}\right),\left(H_{2}\right),\left(H_{3}\right)$, if $u$ is a classical solution of (4), then there exist $K_{i} \in X$, $i=1,2,3,4$, such that, for all $x \in[a, b]$, we have

$$
u(x)=e^{(x-a) M} K_{1}+(x-a) e^{(x-a) M} K_{2}+e^{(b-x) M} K_{3}+(b-x) e^{(b-x) M} K_{4}+F_{0, f}(x),
$$

where $F_{0, f}$ is defined in Theorem 2.6.

Proof. Let $u$ be a classical solution of (4). From Theorem 2.6, there exists a classical solution $F_{0, f}$ of (4)-(BC1). Thus

$$
F_{0, f}(a)=F_{0, f}(b)=F_{0, f}^{\prime \prime}(a)=F_{0, f}^{\prime \prime}(b)=0 .
$$

Set $u_{h}:=u-F_{0, f}$. Then, $u_{h}$ is a classical solution of

$$
u_{h}^{(4)}(x)+2 A u_{h}^{\prime \prime}(x)+A^{2} u_{h}(x)=0, \quad \text { a.e. } x \in(a, b) .
$$

Now, it remains to determine the expression of $u_{h}$. To this end, we have to solve the previous homogeneous equation. For all $x \in[a, b]$, we set

$$
v(x)=u_{h}^{\prime \prime}(x)+A u_{h}(x) .
$$

Since $u_{h} \in W^{4, p}(a, b ; X) \cap L^{p}\left(a, b ; D\left(A^{2}\right)\right)$ with $u_{h}^{\prime \prime} \in L^{p}(a, b ; D(A))$, we deduce that

$$
v \in W^{2, p}(a, b ; X) \cap L^{p}(a, b ; D(A)) .
$$

Then, for a. e. $x \in(a, b)$, we have

$$
v^{\prime \prime}(x)+A v(x)=u_{h}^{(4)}(x)+2 A u_{h}^{\prime \prime}(x)+A^{2} u_{h}(x)=0 .
$$

Using the proof of Theorem 5, p. 173, in [9] or (18) in [10], we obtain that the solution $v$ of the previous homogeneous equation reads as

$$
v(x)=e^{(x-a) M} C_{0}+e^{(b-x) M} C_{1}, \quad \text { for all } x \in[a, b],
$$

where $C_{0}, C_{1} \in X$.

Now, we solve the whole equation

$$
u_{h}^{\prime \prime}(x)+A u_{h}(x)=v(x), \quad \text { for all } x \in[a, b] .
$$

Using again the proof of Theorem 5, p. 173 in [9] or (18) in [10], we obtain that, for all $x \in[a, b]$, the solution $u_{h}$ reads as

$$
u_{h}(x)=e^{(x-a) M} C_{2}+e^{(b-x) M} C_{3}+\frac{1}{2} M^{-1} \int_{a}^{x} e^{(x-s) M} v(s) d s+\frac{1}{2} M^{-1} \int_{x}^{b} e^{(s-x) M} v(s) d s
$$


where $C_{2}, C_{3} \in X$. Then

$$
\begin{aligned}
u_{h}(x)= & e^{(x-a) M} C_{2}+e^{(b-x) M} C_{3}+\frac{1}{2} M^{-1} \int_{a}^{x} e^{(x-s) M}\left(e^{(s-a) M} C_{0}+e^{(b-s) M} C_{1}\right) d s \\
& +\frac{1}{2} M^{-1} \int_{x}^{b} e^{(s-x) M}\left(e^{(s-a) M} C_{0}+e^{(b-s) M} C_{1}\right) d s \\
= & e^{(x-a) M} C_{2}+e^{(b-x) M} C_{3}+\frac{1}{2} M^{-1} \int_{a}^{x} e^{(x-a) M} C_{0} d s+\frac{1}{2} M^{-1} \int_{a}^{x} e^{(x+b-2 s) M} C_{1} d s \\
& +\frac{1}{2} M^{-1} \int_{x}^{b} e^{(2 s-x-a) M} C_{0} d s+\frac{1}{2} M^{-1} \int_{x}^{b} e^{(b-x) M} C_{1} d s \\
= & e^{(x-a) M} C_{2}+(x-a) e^{(x-a) M} \frac{1}{2} M^{-1} C_{0}-\frac{1}{4} M^{-2}\left(e^{(b-x) M}-e^{(x-a) M} e^{(b-a) M}\right) C_{1} \\
& +e^{(b-x) M} C_{3}+\frac{1}{4} M^{-2}\left(e^{(b-x) M} e^{(b-a) M}-e^{(x-a) M}\right) C_{0}+(b-x) e^{(b-x) M} \frac{1}{2} M^{-1} C_{1} \\
= & e^{(x-a) M}\left(C_{2}+\frac{1}{4} M^{-2}\left(e^{(b-a) M} C_{1}-C_{0}\right)\right)+(x-a) e^{(x-a) M} \frac{1}{2} M^{-1} C_{0} \\
& +e^{(b-x) M}\left(C_{3}+\frac{1}{4} M^{-2}\left(e^{(b-a) M} C_{0}-C_{1}\right)\right)+(b-x) e^{(b-x) M} \frac{1}{2} M^{-1} C_{1} .
\end{aligned}
$$

So, we set

$$
K_{1}=C_{2}+\frac{1}{4} M^{-2}\left(e^{(b-a) M} C_{1}-C_{0}\right), \quad K_{2}=\frac{1}{2} M^{-1} C_{0},
$$

and

$$
K_{3}=C_{3}+\frac{1}{4} M^{-2}\left(e^{(b-a) M} C_{0}-C_{1}\right), \quad K_{4}=\frac{1}{2} M^{-1} C_{1} .
$$

We conclude that, for all $x \in[a, b]$, we have

$$
u_{h}(x)=e^{(x-a) M} K_{1}+(x-a) e^{(x-a) M} K_{2}+e^{(b-x) M} K_{3}+(b-x) e^{(b-x) M} K_{4} .
$$

Thus, $u$ satisfies (15), which gives the result.

\subsection{Regularity of polynomial exponential terms}

We state below a result which concerns the regularity of the terms composed by a polynomial function and an analytic semigroup. This result improves the ones obtained in [8], Lemma 3.2 and Proposition 1.

Proposition 4.2. Let $T$ be the infinitesimal generator of a bounded analytic semigroup, $p \in[1,+\infty]$ with $0 \in \rho(T)$. For all $x \in[a, b]$ and $\psi \in X$, we set

$$
v_{\psi}(x)=(x-a) e^{(x-a) T} \psi \quad \text { and } \quad w_{\psi}(x)=(b-x) e^{(b-x) T} \psi .
$$

Then, we have

1. $v_{\psi} \in L^{p}(a, b ; D(T))$.

2. For $n \in \mathbb{N} \backslash\{0,1\}, v_{\psi} \in L^{p}\left(a, b ; D\left(T^{n}\right)\right)$ if and only if $\psi \in D\left(T^{n-2}\right)$ and $T^{n-2} \psi \in(D(T), X)_{\frac{1}{p}, p}$.

3. For $n \in \mathbb{N} \backslash\{0\}$, if $v_{\psi} \in L^{p}\left(a, b ; D\left(T^{n}\right)\right)$ then $v_{\psi} \in W^{n, p}(a, b ; X)$.

Moreover, these three statements hold if we replace $v_{\psi}$ by $w_{\psi}$.

Proof.

1. It is clear that $v_{\psi} \in L^{p}(a, b ; X)$ and from [25], Proposition 2.1.1, statement (iii), p. 35 or [27], equation (6.6), p. 70, we obtain the result. 
2. From [33], Theorem, p. 96, for $\theta \in(0,1)$, we know that

$$
\begin{aligned}
\psi \in\left(X, D\left(T^{n}\right)\right)_{\theta, p} & \Longleftrightarrow \int_{0}^{+\infty}\left\|t^{n(1-\theta)} T^{n} e^{t T} \psi\right\|^{p} \frac{d t}{t}<+\infty \\
& \Longleftrightarrow \int_{0}^{+\infty}\left\|t^{n(1-\theta)-\frac{1}{p}} T^{n} e^{t T} \psi\right\|^{p} d t<+\infty
\end{aligned}
$$

Moreover, for $n \geqslant 2$, we set

$$
\theta=1-\frac{1}{n}-\frac{1}{n p} \in(0,1)
$$

since $n(1-\theta)-\frac{1}{p}=1$, we obtain that

$$
\begin{aligned}
\psi \in\left(X, D\left(T^{n}\right)\right)_{\theta, p} & \Longleftrightarrow \int_{0}^{+\infty}\left\|t T^{n} e^{t T} \psi\right\|^{p} d t<+\infty \\
& \Longleftrightarrow t \longmapsto t T^{n} e^{t T} \psi \in L^{p}(0,+\infty ; X)
\end{aligned}
$$

Replacing $t$ by $x-a$, we have

$$
t \longmapsto t T^{n} e^{t T} \psi \in L^{p}(0,+\infty ; X) \Longleftrightarrow v_{\psi} \in L^{p}\left(a,+\infty ; D\left(T^{n}\right)\right),
$$

and from Lemma 3.2, we deduce that

$$
v_{\psi} \in L^{p}\left(a,+\infty ; D\left(T^{n}\right)\right) \Longleftrightarrow v_{\psi} \in L^{p}\left(a, b ; D\left(T^{n}\right)\right) .
$$

From the reiteration Theorem and Theorem in section 1.14.3 in [33], we obtain

$$
\begin{aligned}
\left(X, D\left(T^{n}\right)\right)_{\theta, p} & =\left(X, D\left(T^{n}\right)\right)_{1-\frac{1}{n}-\frac{1}{n p}, p} \\
& =(X, D(T))_{n-1-\frac{1}{p}, p} \\
& =(X, D(T))_{(n-2)+1-\frac{1}{p}, p}
\end{aligned}
$$

Thus, it follows from (7) that

$$
\psi \in D\left(T^{n-2}\right) \quad \text { and } \quad \psi \in(D(T), X)_{\frac{1}{p}, p} \Longleftrightarrow v_{\psi} \in L^{p}\left(a, b ; D\left(T^{n}\right)\right)
$$

3. For statement 3., we begin by the case $n \geqslant 2$ : if $v_{\psi} \in L^{p}\left(a, b ; D\left(T^{n}\right)\right)$ then from statement 2 ., $\psi \in D\left(T^{n-2}\right)$ and $T^{n-2} \psi \in(D(T), X)_{\frac{1}{p}, p}$, this ensure by Lemma 3.2 that we have

$$
x \longmapsto T^{n-1} e^{(x-a) T} \psi \in L^{p}(a, b ; X) .
$$

We conclude that

$$
v_{\psi}^{(n)}(\cdot)=n T^{n-1} e^{(\cdot-a) T} \psi+T^{n} v_{\psi}(\cdot) \in L^{p}(a, b ; X) .
$$

Now, we consider the case $n=1$. If $v_{\psi} \in L^{p}(a, b ; D(T))$ then we have

$$
v_{\psi}^{\prime}(\cdot)=e^{(\cdot-a) T} \psi+T v_{\psi}(\cdot) \in L^{p}(a, b ; X) .
$$

To prove that these three statements hold if we replace $v_{\psi}$ by $w_{\psi}$, it sufficient to use a variable change: $w_{\psi}(x)=v_{\psi}(b+a-x)$. 


\subsection{Traces result}

Proposition 4.3. Assume that $\left(H_{2}\right),\left(H_{3}\right)$ hold. Let $u$ be a classical solution of (4), then, for $s \in\{a, b\}$, we have

$$
\left\{\begin{array}{lll}
u(s) \in D\left(M^{3}\right) & \text { and } & M^{3} u(s) \in(D(M), X)_{\frac{1}{p}, p}, \\
u^{\prime}(s) \in D\left(M^{2}\right) & \text { and } & M^{2} u^{\prime}(s) \in(D(M), X)_{\frac{1}{p}, p} \\
u^{\prime \prime}(s) \in D(M) & \text { and } & M u^{\prime \prime}(s) \in(D(M), X)_{\frac{1}{p}, p} .
\end{array}\right.
$$

Moreover, we have

$$
\left\{\begin{array}{rlrlrl}
\psi \in D\left(M^{3}\right) \quad \text { and } \quad M^{3} \psi \in(D(M), X)_{\frac{1}{p}, p} & \Longleftrightarrow \psi \in D(A) \quad \text { and } & A \psi \in(D(A), X)_{\frac{1}{2 p}, p}, \\
\psi \in D\left(M^{2}\right) \quad \text { and } \quad M^{2} \psi \in(D(M), X)_{\frac{1}{p}, p} & \Longleftrightarrow \psi \in D(A) \quad \text { and } & A \psi \in(D(A), X)_{\frac{1}{2}+\frac{1}{2 p}, p}, \\
\psi \in D(M) \quad \text { and } \quad M \psi \in(D(M), X)_{\frac{1}{p}, p} & \Longleftrightarrow & & \psi \in(D(A), X)_{\frac{1}{2 p}, p}, \\
\psi \in(D(M), X)_{\frac{1}{p}, p} & \Longleftrightarrow & & \psi \in(D(A), X)_{\frac{1}{2}+\frac{1}{2 p}, p} .
\end{array}\right.
$$

Proof. Since $A^{2}=M^{4}$, we have $u \in W^{4, p}(a, b ; X) \cap L^{p}\left(a, b ; D\left(M^{4}\right)\right)$. Then, from Lemma 3.1, for $s \in\{a, b\}$, we obtain

$$
u(s) \in\left(D\left(M^{4}\right), X\right)_{\frac{1}{4 p}, p}, \quad u^{\prime}(s) \in\left(D\left(M^{4}\right), X\right)_{\frac{1}{4}+\frac{1}{4 p}, p} \quad \text { and } \quad u^{\prime \prime}(s) \in\left(D\left(M^{4}\right), X\right)_{\frac{1}{2}+\frac{1}{4 p}, p},
$$

which leads to (18) by using the reiteration theorem and Theorem in section 1.14 .3 in [33]. Noting that $A=M^{2}$, again by the reiteration theorem and Theorem in section 1.14.3 in [33], we obtain (19).

Note that the result remains true if $\left(H_{3}\right)$ is replaced by a sectoriality assumption on $A$.

\subsection{Functional calculus}

Throughout the paper, we set

$$
c=b-a>0 .
$$

We will need the invertibility of $U$ and $V$ given by

$$
\left\{\begin{aligned}
U & :=I-e^{2 c M}+2 c M e^{c M} \\
V & :=I-e^{2 c M}-2 c M e^{c M} .
\end{aligned}\right.
$$

To this end, we need a result from functional calculus. To state it, we have to set some notations and give some technical lemmas.

Let $\theta \in(0, \pi)$, we denote by $H\left(S_{\theta}\right)$ the space of holomorphic functions on $S_{\theta}$, the sector of angle $\theta$ (see (5)) with values in $\mathbb{C}$. Moreover, we consider the following subspace of $H\left(S_{\theta}\right)$ :

$$
\mathcal{E}_{\infty}\left(S_{\theta}\right):=\left\{f \in H\left(S_{\theta}\right): f=O\left(|z|^{-s}\right)(|z| \rightarrow+\infty) \text { for some } s>0\right\} .
$$

In other words, $\mathcal{E}_{\infty}\left(S_{\theta}\right)$ is the space of polynomial decreasing holomorphic functions at $+\infty$. Let $T$ be an invertible sectorial operator of angle $\theta_{T} \in(0, \pi)$ with bounded inverse. If $f \in \mathcal{E}_{\infty}\left(S_{\theta}\right)$, with $\theta \in\left(\theta_{T}, \pi\right)$, then we can define, by functional calculus, $f(T) \in \mathcal{L}(X)$, see [15], p. 45. In this work, we use functional calculus, as classicaly done, see for instance [16], [18], [21] or [22].

For $z \in \mathbb{C} \backslash \mathbb{R}_{-}$, we set

$$
\left\{\begin{array}{l}
\tilde{u}(z)=1-e^{-2 c \sqrt{z}}-2 c \sqrt{z} e^{-c \sqrt{z}} \\
\tilde{v}(z)=1-e^{-2 c \sqrt{z}}+2 c \sqrt{z} e^{-c \sqrt{z}} .
\end{array}\right.
$$

Note that, we can write formally $U=\tilde{u}(-A)$ and $V=\tilde{v}(-A)$. We set

$$
\mathbb{C}_{+}:=\{z \in \mathbb{C}: \operatorname{Re}(z) \geqslant 0\}
$$

We obtain the following results: 
Lemma 4.4. The complex functions $\tilde{u}$ and $\tilde{v}$, given by $(21)$ do not vanish on $\mathbb{C}_{+} \backslash\{0\}$.

Proof. Let $z \in \mathbb{C} \backslash \mathbb{R}_{-}$such that $\operatorname{Re}(z) \geqslant 0$. Then, we have

$$
\tilde{u}(z)=1-e^{-2 c \sqrt{z}}-2 c \sqrt{z} e^{-c \sqrt{z}}=e^{-c \sqrt{z}}\left(e^{c \sqrt{z}}-e^{-c \sqrt{z}}-2 c \sqrt{z}\right),
$$

and

$$
\tilde{v}(z)=1-e^{-2 c \sqrt{z}}+2 c \sqrt{z} e^{-c \sqrt{z}}=e^{-c \sqrt{z}}\left(e^{c \sqrt{z}}-e^{-c \sqrt{z}}+2 c \sqrt{z}\right) .
$$

Moreover, one has

$$
|\tilde{u}(z)|=\left|e^{-c \sqrt{z}}\right|\left|e^{c \sqrt{z}}-e^{-c \sqrt{z}}-2 c \sqrt{z}\right| \geqslant\left|e^{-c \sqrt{z}}\right||| e^{c \sqrt{z}}-e^{-c \sqrt{z}}|-2| c \sqrt{z}||,
$$

and

$$
|\tilde{v}(z)|=\left|e^{-c \sqrt{z}}\right|\left|e^{c \sqrt{z}}-e^{-c \sqrt{z}}+2 c \sqrt{z}\right| \geqslant\left|e^{-c \sqrt{z}}\right||| e^{c \sqrt{z}}-e^{-c \sqrt{z}}|-2| c \sqrt{z}|| .
$$

Now, we focus on $\left|e^{c \sqrt{z}}-e^{-c \sqrt{z}}\right|$. To this end, we set $\sqrt{z}=\frac{\alpha}{c}+i \frac{\beta}{c}$, where $\alpha, \beta \in \mathbb{R}$. Thus

$$
\left|e^{c \sqrt{z}}-e^{-c \sqrt{z}}\right|^{2}=\left|e^{\alpha+i \beta}-e^{-\alpha-i \beta}\right|^{2}=2(\cosh (2 \alpha)-\cos (2 \beta)),
$$

and

$$
\begin{aligned}
2(\cosh (2 \alpha)-\cos (2 \beta)) & =2 \sum_{n=0}^{+\infty} \frac{2^{2 n}}{(2 n) !}\left(\alpha^{2 n}-(-1)^{n} \beta^{2 n}\right) \\
& =4\left(\alpha^{2}+\beta^{2}\right)+2 \sum_{n=2}^{+\infty} \frac{2^{2 n}}{(2 n) !}\left(\alpha^{2 n}-(-1)^{n} \beta^{2 n}\right) \\
& >4\left(\alpha^{2}+\beta^{2}\right)+2 \sum_{n=2}^{+\infty} \frac{2^{2 n}}{(2 n) !}\left(\alpha^{2 n}-\beta^{2 n}\right) .
\end{aligned}
$$

Since $\operatorname{Re}(z) \geqslant 0$, we deduce that for all $n \in \mathbb{N} \backslash\{0\}$, we have $\alpha^{2 n} \geqslant \beta^{2 n}$. Then, we obtain

$$
4\left(\alpha^{2}+\beta^{2}\right)+2 \sum_{n=2}^{+\infty} \frac{2^{2 n}}{(2 n) !}\left(\alpha^{2 n}-\beta^{2 n}\right) \geqslant 4\left(\alpha^{2}+\beta^{2}\right)=4|\alpha+i \beta|^{2} .
$$

Finally, we have

$$
\left|e^{c \sqrt{z}}-e^{-c \sqrt{z}}\right|^{2}=\left|e^{\alpha+i \beta}-e^{-\alpha-i \beta}\right|^{2}>4|\alpha+i \beta|^{2}=4|c \sqrt{z}|^{2}
$$

hence

$$
\left|e^{c \sqrt{z}}-e^{-c \sqrt{z}}\right|>2|c \sqrt{z}|
$$

This implies that $|\tilde{u}(z)|>0$ and $|\tilde{v}(z)|>0$, for $z \in \mathbb{C}_{+} \backslash\{0\}$.

Proposition 4.5. Assume that $\left(H_{2}\right)$ and $\left(H_{3}\right)$ with $\theta_{A}<\pi / 2$ hold. Then $U$ and $V$, defined by (20), are invertible with bounded inverse.

Proof. From Lemma 4.4, we know that $\tilde{u}$ and $\tilde{v}$, given by $(21)$, do not vanish on $\left(\mathbb{R}_{+} \times \mathbb{R}\right) \backslash\{0\}$. Then, setting $f=\tilde{u}$ or $\tilde{v}$, we have $\frac{1}{f}=1+h$, where $h:=\frac{1-f}{f} \in H\left(S_{\theta_{A}}\right)$. Since $1-f \in \mathcal{E}_{\infty}\left(S_{\theta_{A}}\right)$, there exists $R_{A}>0$ such that, if $z \in S_{\theta_{A}}$ and $|z|>R_{A}$, then $|1-f|\left\langle C|z|^{-\alpha}\right.$ where $\alpha>0, C>0$. Moreover, there exists $R_{0}>0$ such that, for all $z \in S_{\theta_{A}}$ with $|z|>R_{0}$ we have $|1-f(z)|<1 / 2$, we deduce that $|f(z)|>1 / 2$. So, for all $z \in S_{\theta_{A}}$ with $|z|>\max \left(R_{A}, R_{0}\right)$ we obtain $|h(z)| \leq 2 C|z|^{-\alpha}$. It follows that $h \in \mathcal{E}_{\infty}\left(S_{\theta_{A}}\right)$. Indeed, since $\sigma(-A) \subset S_{\theta_{A}}$, from [15], pp. 28 and 45, in this case we can set:

$$
\frac{1}{f}(-A)=I+h(-A) \in \mathcal{L}(X),
$$


and the invertibility of $f(-A)=U$ or $V$ is obtained by writing

$$
\frac{1}{f}(-A) f(-A)=\left(\frac{1}{f} \times f\right)(-A)=1(-A)=I
$$

and similarly, $f(-A) \frac{1}{f}(-A)=I$.

\section{Proof of Theorem 2.8}

Note that from Proposition 4.3, in Theorem 2.8, we just have to prove the reciprocal implications. For each boundary conditions, the proof of Theorem 2.8 is divided in two steps. First, using the representation formula obtained in Proposition 4.1, we show the uniqueness of the classical solution. Then, in a second step, we state that the solution given by the representation formula is a classical solution.

\subsection{Proof of statement 1. (Boundary Conditions (BC2))}

Assume that $\left(H_{1}\right),\left(H_{2}\right),\left(H_{3}\right)$ and $(12)$ hold. First of all, we will show that the solution of problem (4)-(BC2) is unique by determining constants $K_{i}, i=1,2,3,4$ of the representation formula (15). Then, we will prove that this formula is a classical solution.

First step: Uniqueness.

If $u$ is a classical solution of problem (4)-(BC2), then from Proposition 4.1, $u$ reads as

$$
u(x)=e^{(x-a) M} K_{1}+(x-a) e^{(x-a) M} K_{2}+e^{(b-x) M} K_{3}+(b-x) e^{(b-x) M} K_{4}+F_{0, f}(x) .
$$

To conclude, it is sufficient to show that $K_{1}, K_{2}, K_{3}$ and $K_{4}$ are uniquely determined.

Since $F_{0, f}$ satisfies (16), by making explicit the boundary conditions with $M^{-1} u(x), M^{-1} u^{\prime}(x)$ and $M^{-1} u^{\prime \prime}(x)$, we obtain the following system:

$$
\left\{\begin{aligned}
K_{1}+M^{-1} K_{2}-e^{c M} K_{3}-\left(c I+M^{-1}\right) e^{c M} K_{4} & =M^{-1}\left(\varphi_{1}-F_{0, f}^{\prime}(a)\right) \\
e^{c M} K_{1}+\left(c I+M^{-1}\right) e^{c M} K_{2}-K_{3}-M^{-1} K_{4} & =M^{-1}\left(\varphi_{2}-F_{0, f}^{\prime}(b)\right) \\
2 K_{2}+2 e^{c M} K_{4} & =M^{-1} \varphi_{3} \\
2 e^{c M} K_{2}+2 K_{4} & =M^{-1} \varphi_{4} .
\end{aligned}\right.
$$

Note that we have considered $M^{-1} u(x), M^{-1} u^{\prime}(x)$ and $M^{-1} u^{\prime \prime}(x)$, because we do not know if $K_{i} \in D(M), i=1,2,3,4$. We deduce that this system can be written as two uncoupled sub-systems:

$$
\left\{\begin{array}{l}
K_{1}-e^{c M} K_{3}=M^{-1}\left(\varphi_{1}-F_{0, f}^{\prime}(a)-K_{2}\right)+\left(c I+M^{-1}\right) e^{c M} K_{4} \\
e^{c M} K_{1}-K_{3}=M^{-1}\left(\varphi_{2}-F_{0, f}^{\prime}(b)+K_{4}\right)-\left(c I+M^{-1}\right) e^{c M} K_{2}
\end{array}\right.
$$

and

$$
\left\{\begin{aligned}
K_{2}+e^{c M} K_{4} & =\frac{1}{2} M^{-1} \varphi_{3} \\
e^{c M} K_{2}+K_{4} & =\frac{1}{2} M^{-1} \varphi_{4}
\end{aligned}\right.
$$

which we have to solve. We begin by system (23). We calculate the determinant of the associated matrix called $\Lambda_{1}$. Hence

$$
\operatorname{det}\left(\Lambda_{1}\right)=I-e^{2 c M},
$$


which is invertible with bounded inverse from [25], Proposition 2.3.6, p. 60. Thus

$$
\left\{\begin{array}{l}
K_{2}=\frac{1}{2} M^{-1}\left(I-e^{2 c M}\right)^{-1}\left(\varphi_{3}-e^{c M} \varphi_{4}\right) \\
K_{4}=\frac{1}{2} M^{-1}\left(I-e^{2 c M}\right)^{-1}\left(\varphi_{4}-e^{c M} \varphi_{3}\right)
\end{array}\right.
$$

To determine $K_{1}$ and $K_{3}$ (as function of $K_{2}$ and $K_{4}$ ), it is sufficient to remark that system (22) reads as

$$
\left\{\begin{array}{l}
K_{1}-e^{c M} K_{3}=M^{-1}\left(\varphi_{1}-F_{0, f}^{\prime}(a)-K_{2}\right)+\left(c I+M^{-1}\right) e^{c M} K_{4} \\
e^{c M} K_{1}-K_{3}=M^{-1}\left(\varphi_{2}-F_{0, f}^{\prime}(b)+K_{4}\right)-\left(c I+M^{-1}\right) e^{c M} K_{2} .
\end{array}\right.
$$

Then we compute the determinant of the associated matrix called $\Lambda_{2}$.

$$
\operatorname{det}\left(\Lambda_{2}\right)=-\left(I-e^{2 c M}\right),
$$

which is invertible with bounded inverse from [25], Proposition 2.3.6, p. 60. Thus $K_{1}$ and $K_{3}$ are uniquely determined. Moreover, we have

$$
\left\{\begin{array}{l}
K_{1}=M^{-1}\left(\varphi_{1}-F_{0, f}^{\prime}(a)-K_{2}\right)+\left(c I+M^{-1}\right) e^{c M} K_{4}+e^{c M} K_{3} \\
K_{3}=-M^{-1}\left(\varphi_{2}-F_{0, f}^{\prime}(b)+K_{4}\right)+\left(c I+M^{-1}\right) e^{c M} K_{2}+e^{c M} K_{1}
\end{array}\right.
$$

where $e^{c M} K_{1}$ and $e^{c M} K_{3}$ are regular terms, see (26) below.

Second step: Existence.

From the previous step, if the classical solution exists, it is unique and is given by the representation formula (15). Note that since $F_{0, f} \in W^{4, p}(a, b ; X) \cap L^{p}\left(a, b ; D\left(A^{2}\right)\right)$, then from Lemma 3.1, (16), we have

$$
F_{0, f}^{\prime}(a), F_{0, f}^{\prime}(b) \in D\left(M^{2}\right) \quad \text { and } \quad M^{2} F_{0, f}^{\prime}(a), M^{2} F_{0, f}^{\prime}(b) \in(D(M), X)_{\frac{1}{p}, p} .
$$

We have to make explicit constants $K_{i}, i=1,2,3,4$ and to determine their regularity. For $c>0$ and $\psi \in X$, one has

$$
e^{c M} \psi \in D\left(M^{\infty}\right):=\bigcap_{k \geqslant 0} D\left(M^{k}\right)
$$

Thus, from Lemma 3.3, we have

$$
\left(I \pm e^{2 c M}\right)^{-1}=I+R_{ \pm}, \quad \text { where } \quad R_{ \pm}(X) \subset D\left(M^{\infty}\right) \quad \text { and } \quad R_{ \pm} M=M R_{ \pm} .
$$

Then, from (24), (26) and Lemma 3.3, there exist $R_{i} \in D\left(M^{\infty}\right), i=1,2,3,4$, such that

$$
\left\{\begin{aligned}
K_{2} & =\frac{1}{2} M^{-1} \varphi_{3}+R_{2} \\
K_{4} & =\frac{1}{2} M^{-1} \varphi_{4}+R_{4} \\
K_{1} & =M^{-1}\left(\varphi_{1}-F_{0, f}^{\prime}(a)-K_{2}\right)+R_{1} \\
K_{3} & =-M^{-1}\left(\varphi_{2}-F_{0, f}^{\prime}(b)+K_{4}\right)+R_{3} .
\end{aligned}\right.
$$

So, from (12) and (25), we have

$$
K_{2}, K_{4} \in D\left(M^{2}\right) \quad \text { and } \quad M^{2} K_{2}, M^{2} K_{4} \in(D(M), X)_{\frac{1}{p}, p},
$$

hence, we deduce that

$$
K_{1}, K_{3} \in D\left(M^{3}\right) \quad \text { and } \quad M^{3} K_{1}, M^{3} K_{3} \in(D(M), X)_{\frac{1}{p}, p} .
$$


Thus, from Lemma 3.2 , we obtain that $u_{M}: x \longmapsto e^{(x-a) M} K_{1}+e^{(b-x) M} K_{3}$, satisfies

$$
u_{M} \in W^{4, p}(a, b ; X) \cap L^{p}\left(a, b ; D\left(A^{2}\right)\right) \quad \text { and } \quad u_{M}^{\prime \prime} \in L^{p}(a, b ; D(A)) .
$$

From Proposition 4.2 , we deduce that $v_{K_{2}}: x \longmapsto(x-a) e^{(x-a) M} K_{2}$ satisfies

$$
v_{K_{2}} \in W^{4, p}(a, b ; X) \cap L^{p}\left(a, b ; D\left(A^{2}\right)\right) \quad \text { and } \quad v_{K_{2}}^{\prime \prime} \in L^{p}(a, b ; D(A)) .
$$

In the same way, $v_{K_{4}}: x \longmapsto(b-x) e^{(b-x) M} K_{4}$ satisfies

$$
v_{K_{4}} \in W^{4, p}(a, b ; X) \cap L^{p}\left(a, b ; D\left(A^{2}\right)\right) \quad \text { and } \quad v_{K_{4}}^{\prime \prime} \in L^{p}(a, b ; D(A)) .
$$

Since $F_{0, f}$ is a classical solution of (4), from (27), (28) and (29), $u$ is a classical solution of problem (4)-(BC2).

\subsection{Proof of statement 2. (Boundary Conditions (BC3))}

Now, we assume that $\left(H_{1}\right),\left(H_{2}\right)$ and $\left(H_{3}\right)$ with $\theta_{A}<\pi / 2$ hold. For the statement 1 ., the representation formula was easily obtained by taking into account the boundary conditions. Nevertheless, for the following statements, to build the representation formula, we need the invertibility of determinants operators, defined by (20). This invertibility has been proved in Proposition 4.5 .

Proof of 2. of Theorem 2.8. Assume that (13) holds. We will show that there exists a unique classical solution of (4)-(BC3). The proof is divided in two step. First, we prove the uniqueness of the solution by determining constants $K_{i}, i=1,2,3,4$, of the representation formula (15). Then, we show that the formula obtained is a classical solution.

First step: Uniqueness.

If $u$ is a classical solution of problem (4)-(BC3), then from Proposition 4.1, the solution $u$ reads as

$$
u(x)=e^{(x-a) M} K_{1}+(x-a) e^{(x-a) M} K_{2}+e^{(b-x) M} K_{3}+(b-x) e^{(b-x) M} K_{4}+F_{0, f}(x) .
$$

To simplify our computations, we set

$$
\alpha_{1}:=\frac{K_{1}-K_{3}}{2}, \quad \alpha_{2}:=\frac{K_{2}-K_{4}}{2}, \quad \alpha_{3}:=\frac{K_{1}+K_{3}}{2} \quad \text { and } \quad \alpha_{4}:=\frac{K_{2}+K_{4}}{2} .
$$

Then, $u$ is given by

$$
\begin{aligned}
u(x)= & \left(e^{(x-a) M}-e^{(b-x) M}\right) \alpha_{1}+\left((x-a) e^{(x-a) M}-(b-x) e^{(b-x) M}\right) \alpha_{2} \\
& +\left(e^{(x-a) M}+e^{(b-x) M}\right) \alpha_{3}+\left((x-a) e^{(x-a) M}+(b-x) e^{(b-x) M}\right) \alpha_{4}+F_{0, f}(x) .
\end{aligned}
$$

As $F_{0, f}$ satisfies (16), to make explicit the boundary conditions, we consider $M^{-1} u(x)$ and $M^{-1} u^{\prime}(x)$, since we do not know if $\alpha_{1}, \alpha_{3} \in D(M)$. Therefore, we obtain the following equations:

$$
\begin{aligned}
\left(I-e^{c M}\right) M^{-1} \alpha_{1}-c e^{c M} M^{-1} \alpha_{2}+\left(I+e^{c M}\right) M^{-1} \alpha_{3}+c e^{c M} M^{-1} \alpha_{4}=M^{-1} \varphi_{1} \\
-\left(I-e^{c M}\right) M^{-1} \alpha_{1}+c e^{c M} M^{-1} \alpha_{2}+\left(I+e^{c M}\right) M^{-1} \alpha_{3}+c e^{c M} M^{-1} \alpha_{4}=M^{-1} \varphi_{2} \\
\quad\left(I+e^{c M}\right) \alpha_{1}+\left(I+(c M+I) e^{c M}\right) M^{-1} \alpha_{2} \\
+\left(I-e^{c M}\right) \alpha_{3}+\left(I-(c M+I) e^{c M}\right) M^{-1} \alpha_{4}=M^{-1}\left(\varphi_{3}-F_{0, f}^{\prime}(a)\right) \\
\quad\left(I+e^{c M}\right) \alpha_{1}+\left(I+(c M+I) e^{c M}\right) M^{-1} \alpha_{2} \\
-\left(I-e^{c M}\right) \alpha_{3}-\left(I-(c M+I) e^{c M}\right) M^{-1} \alpha_{4}=M^{-1}\left(\varphi_{4}-F_{0, f}^{\prime}(b)\right)
\end{aligned}
$$


Summing and subtracting (32) with (33) and summing and subtracting (34) with (35), we obtain the following system

$$
\left\{\begin{aligned}
\left(I+e^{c M}\right) \alpha_{3}+c e^{c M} \alpha_{4} & =\frac{\varphi_{1}+\varphi_{2}}{2} \\
\left(I-e^{c M}\right) \alpha_{3}+\left(I-(c M+I) e^{c M}\right) M^{-1} \alpha_{4} & =M^{-1} \tilde{\varphi}_{1} \\
-\left(I-e^{c M}\right) \alpha_{1}+c e^{c M} \alpha_{2} & =\frac{\varphi_{2}-\varphi_{1}}{2} \\
\left(I+e^{c M}\right) \alpha_{1}+\left(I+(c M+I) e^{c M}\right) M^{-1} \alpha_{2} & =M^{-1} \tilde{\varphi}_{2}
\end{aligned}\right.
$$

where

$$
\tilde{\varphi}_{1}:=\frac{\varphi_{3}-\varphi_{4}+F_{0, f}^{\prime}(b)-F_{0, f}^{\prime}(a)}{2} \quad \text { and } \quad \tilde{\varphi}_{2}:=\frac{\varphi_{3}+\varphi_{4}-F_{0, f}^{\prime}(a)-F_{0, f}^{\prime}(b)}{2} .
$$

We deduce that this system can be written as two uncoupled sub-systems:

$$
\left\{\begin{aligned}
\left(I+e^{c M}\right) \alpha_{3}+c e^{c M} \alpha_{4} & =\frac{\varphi_{1}+\varphi_{2}}{2} \\
\left(I-e^{c M}\right) \alpha_{3}+\left(I-(c M+I) e^{c M}\right) M^{-1} \alpha_{4} & =M^{-1} \tilde{\varphi}_{1}
\end{aligned}\right.
$$

and

$$
\left\{\begin{aligned}
-\left(I-e^{c M}\right) \alpha_{1}+c e^{c M} \alpha_{2} & =\frac{\varphi_{2}-\varphi_{1}}{2} \\
\left(I+e^{c M}\right) \alpha_{1}+\left(I+(c M+I) e^{c M}\right) M^{-1} \alpha_{2} & =M^{-1} \tilde{\varphi_{2}},
\end{aligned}\right.
$$

which we have to solve. We begin by system (37). By summing both lines, we obtain

$$
\left\{\begin{aligned}
\left(I+e^{c M}\right) \alpha_{3}+c e^{c M} \alpha_{4} & =\frac{\varphi_{1}+\varphi_{2}}{2} \\
2 \alpha_{3}+\left(I-e^{c M}\right) M^{-1} \alpha_{4} & =M^{-1} \tilde{\varphi}_{1}+\frac{\varphi_{1}+\varphi_{2}}{2} .
\end{aligned}\right.
$$

Then, we calculate the determinant of the associated matrix $\Lambda_{1}$,

$$
\begin{aligned}
\operatorname{det}\left(\Lambda_{1}\right) & =\left(I+e^{c M}\right)\left(I-e^{c M}\right) M^{-1}-2 c e^{c M} \\
& =M^{-1}\left(I-e^{2 c M}-2 c M e^{c M}\right) \\
& =M^{-1} V
\end{aligned}
$$

where $V$ is defined by $(20)$.

Now, we consider system (38). By subtracting the first line to the second, we obtain

$$
\left\{\begin{aligned}
-\left(I-e^{c M}\right) \alpha_{1}+c e^{c M} \alpha_{2} & =\frac{\varphi_{2}-\varphi_{1}}{2} \\
2 \alpha_{1}+\left(I+e^{c M}\right) M^{-1} \alpha_{2} & =M^{-1} \tilde{\varphi}_{2}-\frac{\varphi_{2}-\varphi_{1}}{2} .
\end{aligned}\right.
$$

Then, we calculate the determinant of the associated matrix $\Lambda_{2}$,

$$
\begin{aligned}
\operatorname{det}\left(\Lambda_{2}\right) & =-\left(I-e^{c M}\right)\left(I+e^{c M}\right) M^{-1}-2 c e^{c M} \\
& =-M^{-1}\left(I-e^{2 c M}+2 c M e^{c M}\right) \\
& =-M^{-1} U,
\end{aligned}
$$


where $U$ is defined by $(20)$.

From Proposition 4.5, $U$ and $V$ are invertible with bounded inverse. Thus, if $u$ is a classical solution of (4)-(BC3), then $u$ is uniquely determined by (31), where $\alpha_{i}, i=1,2,3,4$ (which the explicit expression is given in the second step) are the unique solutions of systems (39) and (40).

\section{Second step: Existence.}

From the previous step, if the classical solution $u$ exists, it is unique and it is given by (31), (39) and (40). Now we have to make explicit constants $\alpha_{i}$ and $K_{i}, i=1,2,3,4$ to determine their regularity. From (36) and (40), we have

$$
\left\{\begin{aligned}
\alpha_{1}= & \frac{1}{2} U^{-1}\left(\left(I+(I+c M) e^{c M}\right)\left(\varphi_{1}-\varphi_{2}\right)+c e^{c M}\left(\varphi_{3}+\varphi_{4}\right)\right) \\
& \quad-\frac{c}{2} U^{-1} e^{c M}\left(F_{0, f}^{\prime}(a)+F_{0, f}^{\prime}(b)\right) \\
\alpha_{2}= & \frac{1}{2} U^{-1}\left(\left(I+e^{c M}\right) M\left(\varphi_{2}-\varphi_{1}\right)+\left(I-e^{c M}\right)\left(\varphi_{3}+\varphi_{4}\right)\right) \\
& \quad-\frac{1}{2} U^{-1}\left(I-e^{c M}\right)\left(F_{0, f}^{\prime}(a)+F_{0, f}^{\prime}(b)\right)
\end{aligned}\right.
$$

In the same way, from (36) and (39), we have

$$
\left\{\begin{aligned}
\alpha_{3}= & \frac{1}{2} V^{-1}\left(\left(I-(I+c M) e^{c M}\right)\left(\varphi_{1}+\varphi_{2}\right)-c e^{c M}\left(\varphi_{3}-\varphi_{4}\right)\right) \\
& +\frac{c}{2} V^{-1} e^{c M}\left(F_{0, f}^{\prime}(a)-F_{0, f}^{\prime}(b)\right) \\
\alpha_{4}= & -\frac{1}{2} V^{-1}\left(\left(I-e^{c M}\right) M\left(\varphi_{1}+\varphi_{2}\right)-\left(I+e^{c M}\right)\left(\varphi_{3}-\varphi_{4}\right)\right) \\
& -\frac{1}{2} V^{-1}\left(I+e^{c M}\right)\left(F_{0, f}^{\prime}(a)-F_{0, f}^{\prime}(b)\right) .
\end{aligned}\right.
$$

From (26), there exist $B_{U} \in D\left(M^{\infty}\right)$ and $B_{V} \in D\left(M^{\infty}\right)$ such that $U=I+B_{U}$ and $V=I+B_{V}$. Then, from Lemma 3.3, we have

$$
U^{-1}=I+R_{U} \quad \text { and } \quad V^{-1}=I+R_{V} .
$$

with

$$
R_{U}(X), R_{V}(X) \subset D\left(M^{\infty}\right), \quad R_{U} M=M R_{U} \quad \text { and } \quad R_{V} M=M R_{V} .
$$

We deduce that there exist $R_{i} \in D\left(M^{\infty}\right), i=1,2,3,4$, such that

$$
\left\{\begin{aligned}
\alpha_{1} & =\frac{1}{2}\left(\varphi_{1}-\varphi_{2}\right)+R_{1} \\
\alpha_{2} & =\frac{1}{2}\left(M\left(\varphi_{2}-\varphi_{1}\right)+\left(\varphi_{3}+\varphi_{4}\right)-F_{0, f}^{\prime}(a)-F_{0, f}^{\prime}(b)\right)+R_{2} \\
\alpha_{3} & =\frac{1}{2}\left(\varphi_{1}+\varphi_{2}\right)+R_{3} \\
\alpha_{4} & =-\frac{1}{2}\left(M\left(\varphi_{1}+\varphi_{2}\right)-\left(\varphi_{3}-\varphi_{4}\right)+F_{0, f}^{\prime}(a)-F_{0, f}^{\prime}(b)\right)+R_{4} .
\end{aligned}\right.
$$

From (25) and (30), there exist $\mathcal{R}_{1}, \mathcal{R}_{3} \in D\left(M^{3}\right)$ and $\mathcal{R}_{2}, \mathcal{R}_{4} \in D\left(M^{2}\right)$ with

$$
M^{3} \mathcal{R}_{1}, M^{2} \mathcal{R}_{2}, M^{3} \mathcal{R}_{3}, M^{2} \mathcal{R}_{4} \in(D(M), X)_{\frac{1}{p}, p},
$$

such that

$$
\left\{\begin{array}{l}
K_{1}=\varphi_{1}+\mathcal{R}_{1} \\
K_{2}=\varphi_{3}-M \varphi_{1}+\mathcal{R}_{2} \\
K_{3}=\varphi_{2}+\mathcal{R}_{3} \\
K_{4}=-M \varphi_{2}-\varphi_{4}+\mathcal{R}_{4} .
\end{array}\right.
$$


Then, from (13), since $\varphi_{1}, \varphi_{2} \in D\left(M^{3}\right)$ and $M^{3} \varphi_{1}, M^{3} \varphi_{2} \in(D(M), X)_{\frac{1}{p}, p}$, we have

$$
K_{1}=\varphi_{1}+\mathcal{R}_{1} \in D\left(M^{3}\right) \quad \text { with } \quad M^{3} K_{1} \in(D(M), X)_{\frac{1}{p}, p},
$$

and

$$
K_{3}=\varphi_{2}+\mathcal{R}_{3} \in D\left(M^{3}\right) \quad \text { with } \quad M^{3} K_{3} \in(D(M), X)_{\frac{1}{p}, p} .
$$

From Lemma 3.2, $u_{M}: x \longmapsto e^{(x-a) M} K_{1}+e^{(b-x) M} K_{3}$ satisfies

$$
u_{M} \in W^{4, p}(a, b ; X) \cap L^{p}\left(a, b ; D\left(A^{2}\right)\right) \quad \text { with } \quad u_{M}^{\prime \prime} \in L^{p}(a, b ; D(A)) .
$$

In the same way, from (13), since $\varphi_{1}, \varphi_{2} \in D\left(M^{3}\right)$ and $\varphi_{3}, \varphi_{4} \in D\left(M^{2}\right)$ such that

$$
M^{3} \varphi_{1}, M^{3} \varphi_{2}, M^{2} \varphi_{3}, M^{2} \varphi_{4} \in(D(M), X)_{\frac{1}{p}, p},
$$

we have

$$
K_{2}=\varphi_{3}-M \varphi_{1}+\mathcal{R}_{2} \in D\left(M^{2}\right) \quad \text { with } \quad M^{2} K_{2} \in(D(M), X)_{\frac{1}{p}, p},
$$

and

$$
K_{4}=-M \varphi_{2}-\varphi_{4}+\mathcal{R}_{4} \in D\left(M^{2}\right) \quad \text { with } \quad M^{2} K_{4} \in(D(M), X)_{\frac{1}{p}, p} .
$$

From Proposition 4.2, $v_{K_{2}}: x \longmapsto(x-a) e^{(x-a) M} K_{2}$ satisfies

$$
v_{K_{2}} \in W^{4, p}(a, b ; X) \cap L^{p}\left(a, b ; D\left(A^{2}\right)\right) \quad \text { and } \quad v_{K_{2}}^{\prime \prime} \in L^{p}(a, b ; D(A)),
$$

moreover, $v_{K_{4}}: x \longmapsto(b-x) e^{(b-x) M} K_{4}$ satisfies

$$
v_{K_{4}} \in W^{4, p}(a, b ; X) \cap L^{p}\left(a, b ; D\left(A^{2}\right)\right) \quad \text { and } \quad v_{K_{4}}^{\prime \prime} \in L^{p}(a, b ; D(A)) .
$$

Since $F_{0, f}$ is a classical solution of (4), from (41), (42) and (43), we deduce that $u$ is a classical solution of (4)-(BC3).

\subsection{Proof of statement 3. (Boundary Conditions (BC4))}

We proceed as in the previous proof and we also assume that $\left(H_{1}\right),\left(H_{2}\right)$ and $\left(H_{3}\right)$ with $\theta_{A}<\pi / 2$ hold. We only point out the differences between the two proofs.

Proof. Assume that (14) holds.

First step: Uniqueness.

If $u$ is a classical solution of (4)-(BC4), then from Proposition 4.1, $u$ is given by (31). Since $F_{0, f}$ satisfies (16), by using $M^{-2} u^{\prime}(x)$ and $M^{-2} u^{\prime \prime}(x)$ and following the same computations as done before to make explicit the boundary conditions, we obtain the two following uncoupled sub-systems:

$$
\left\{\begin{aligned}
\left(I+e^{c M}\right) \alpha_{1}+\left(I+(I+c M) e^{c M}\right) M^{-1} \alpha_{2} & =M^{-1} \tilde{\varphi}_{1} \\
2 \alpha_{1}+\left(3 I-e^{c M}\right) M^{-1} \alpha_{2} & =M^{-1}\left(M^{-1}\left(\frac{\varphi_{3}-\varphi_{4}}{2}\right)+\tilde{\varphi}_{1}\right),
\end{aligned}\right.
$$

and

$$
\left\{\begin{aligned}
\left(I-e^{c M}\right) \alpha_{3}+\left(I-(I+c M) e^{c M}\right) M^{-1} \alpha_{4} & =M^{-1} \tilde{\varphi}_{2} \\
2 \alpha_{3}+\left(3 I+e^{c M}\right) M^{-1} \alpha_{4} & =M^{-1}\left(M^{-1}\left(\frac{\varphi_{3}+\varphi_{4}}{2}\right)+\tilde{\varphi}_{2}\right),
\end{aligned}\right.
$$


where

$$
\tilde{\varphi}_{1}:=\frac{\varphi_{1}+\varphi_{2}-F_{0, f}^{\prime}(a)-F_{0, f}^{\prime}(b)}{2} \quad \text { and } \quad \tilde{\varphi}_{2}:=\frac{\varphi_{1}-\varphi_{2}+F_{0, f}^{\prime}(b)-F_{0, f}^{\prime}(a)}{2} .
$$

We calculate the determinant of the associated matrix of system (44) called $\Lambda_{1}$,

$$
\begin{aligned}
\operatorname{det}\left(\Lambda_{1}\right) & =\left(I+e^{c M}\right)\left(3 I-e^{c M}\right) M^{-1}-2\left(I+(I+c M) e^{c M}\right) M^{-1} \\
& =M^{-1}\left(I-e^{2 c M}-2 c M e^{c M}\right) \\
& =M^{-1} V
\end{aligned}
$$

where $V$ is defined by (20). Then, we do the same for $\Lambda_{2}$, the associated matrix of system (45),

$$
\begin{aligned}
\operatorname{det}\left(\Lambda_{2}\right) & =\left(I-e^{c M}\right)\left(3 I+e^{c M}\right) M^{-1}-2\left(I-(I+c M) e^{c M}\right) M^{-1} \\
& =M^{-1}\left(I-e^{2 c M}+2 c M e^{c M}\right) \\
& =M^{-1} U
\end{aligned}
$$

where $U$ is defined by $(20)$.

From Proposition 4.5, $U$ and $V$ are invertible with bounded inverse. Thus, from (44), (45) and (46), we obtain

$$
\left\{\begin{aligned}
\alpha_{1}= & \frac{1}{2} M^{-1} V^{-1}\left(\left(3 I-e^{c M}\right)\left(\varphi_{1}+\varphi_{2}\right)-\left(M^{-1}\left(\varphi_{3}-\varphi_{4}\right)+\varphi_{1}+\varphi_{2}\right)\right) \\
& -\frac{1}{2} M^{-1} V^{-1}(I+c M)\left(M^{-1}\left(\varphi_{3}-\varphi_{4}\right)+\varphi_{1}+\varphi_{2}\right) e^{c M} \\
& -\frac{1}{2} M^{-1} V^{-1}\left(3 I-e^{c M}-\left(I+(I+c M) e^{c M}\right)\right)\left(F_{0, f}^{\prime}(a)+F_{0, f}^{\prime}(b)\right) \\
\alpha_{2}= & -\frac{1}{2} V^{-1}\left(2\left(\varphi_{1}+\varphi_{2}\right)-\left(I+e^{c M}\right)\left(M^{-1}\left(\varphi_{3}-\varphi_{4}\right)+\varphi_{1}+\varphi_{2}\right)\right) \\
& +\frac{1}{2} V^{-1}\left(I-e^{c M}\right)\left(F_{0, f}^{\prime}(a)+F_{0, f}^{\prime}(b)\right)
\end{aligned}\right.
$$

and

$$
\left\{\begin{aligned}
\alpha_{3}= & \frac{1}{2} M^{-1} U^{-1}\left(\left(2 I+(2 I+c M) e^{c M}\right)\left(\varphi_{1}-\varphi_{2}\right)-M^{-1}\left(\varphi_{3}+\varphi_{4}\right)\right) \\
+ & \frac{1}{2} M^{-2} U^{-1}(I+c M)\left(\varphi_{3}+\varphi_{4}\right) e^{c M} \\
+ & \frac{1}{2} M^{-1} U^{-1}\left(2 I+(2 I+c M) e^{c M}\right)\left(F_{0, f}^{\prime}(b)-F_{0, f}^{\prime}(a)\right) \\
\alpha_{4}= & -\frac{1}{2} U^{-1}\left(\left(I+e^{c M}\right)\left(\varphi_{1}-\varphi_{2}\right)-\left(I-e^{c M}\right) M^{-1}\left(\varphi_{3}+\varphi_{4}\right)\right) \\
& -\frac{1}{2} U^{-1}\left(I+e^{c M}\right)\left(F_{0, f}^{\prime}(b)-F_{0, f}^{\prime}(a)\right)
\end{aligned}\right.
$$

Therefore, $u$ is a classical solution of (4)-(BC4), then $u$ is uniquely determined by (31), where coefficients $\alpha_{i}, i=1,2,3,4$, are given by (47) and (48).

\section{Second step: Existence.}

From the previous step, if the solution $u$ exists, it is unique and it is given by (31), (44) and (45). From (26), (30) and Lemma 3.3, we deduce that there exist $R_{i} \in D\left(M^{\infty}\right), i=1,2,3,4$, such that

$$
\left\{\begin{array}{l}
K_{1}=2 M^{-1} \varphi_{1}-M^{-2} \varphi_{3}-2 M^{-1} F_{0, f}^{\prime}(a)+R_{1} \\
K_{2}=-\varphi_{1}+M^{-1} \varphi_{3}+F_{0, f}^{\prime}(a)+R_{2} \\
K_{3}=-2 M^{-1} \varphi_{2}-M^{-2} \varphi_{4}+2 M^{-1} F_{0, f}^{\prime}(b)+R_{3} \\
K_{4}=\varphi_{2}+M^{-1} \varphi_{4}-F_{0, f}^{\prime}(b)+R_{4} .
\end{array}\right.
$$


Then, from (14) and (25), we have

$$
K_{1}, K_{3} \in D\left(M^{3}\right) \quad \text { and } \quad M^{3} K_{1}, M^{3} K_{3} \in(D(M), X)_{\frac{1}{p}, p} .
$$

From Lemma 3.2, $u_{1}: x \longmapsto e^{(x-a) M} K_{1}$ and $u_{3}: x \longmapsto e^{(b-x) M} K_{3}$, satisfy

$$
u_{1}, u_{3} \in W^{4, p}(a, b ; X) \cap L^{p}\left(a, b ; D\left(A^{2}\right)\right) \quad \text { and } \quad u_{1}^{\prime \prime}, u_{3}^{\prime \prime} \in L^{p}(a, b ; D(A)) .
$$

In the same way, from (14) and (25), we deduce

$$
K_{2}, K_{4} \in D\left(M^{2}\right) \quad \text { and } \quad M^{2} K_{2}, M^{2} K_{4} \in(D(M), X)_{\frac{1}{p}, p} .
$$

From Proposition 4.2, $v_{2}: x \longmapsto(x-a) e^{(x-a) M} K_{2}$ and $v_{4}: x \longmapsto(b-x) e^{(b-x) M} K_{4}$ satisfy

$$
v_{2}, v_{4} \in W^{4, p}(a, b ; X) \cap L^{p}\left(a, b ; D\left(A^{2}\right)\right) \quad \text { and } \quad v_{2}^{\prime \prime}, v_{4}^{\prime \prime} \in L^{p}(a, b ; D(A)) .
$$

Therefore, since $F_{0, f}$ is a classical solution of (4), from (49) and (50), we deduce that $u$ is a classical solution of (4)-(BC4).

\section{Conclusion}

In this work, the biharmonic equation with boundary conditions is studied. This model which is described by a fourth order operational equation, within a cylindrical $n$-dimensional spatial region $\Omega$ of $\mathbb{R}^{n}$ is analysed using mathematical tools borrowed from interpolation spaces and Dore-Venni sums theory. The main result could be applied, for instance, to study a transmission problem in two juxtaposed habitats. In the next important step, we will focus on the nonlinear case.

\section{Acknowledgments}

This research is supported by CIFRE contract 2014/1307 with Qualiom Eco company.

The author would like to thank the referee for the valuable and useful comments and corrections which help to improve this paper.

\section{References}

[1] J. Bourgain, "Some remarks on Banach spaces in which martingale difference sequences are unconditional", Ark. Mat., vol. 21,1983, pp. 163-168.

[2] D.L. Burkholder, "A geometrical characterisation of Banach spaces in which martingale difference sequences are unconditional", Ann. Probab., vol. 9, 1981, pp. 997-1011.

[3] F. Cakoni, G. C. Hsiao \& W. L. Wendland, "On the boundary integral equation methodfor a mixed boundary value problem of thebiharmonic equation", Complex variables, Vol. 50, No. 7-11, 2005, pp. 681-696.

[4] D.S. Cohen \& J.D. Murray, "A generalized diffusion model for growth and dispersal in population", Journal of Mathematical Biology, 12, Springer-Verlag, 1981, pp. 237-249.

[5] M. Costabel, E. Stephan \& W. L. Wengland, "On boundary integral equations of the first kind for the bi-Laplacian in a polygonal plane domain", Annali della Scuola Normale Superiore di Pisa, Classe di Scienze, 4 e serie, tome 10, no 2, 1983, pp. 197-241.

[6] G. Da Prato \& P. Grisvard, "Sommes d'opérateurs linéaires et équations différentielles opérationnelle", J. Math. pures et appl., 54, 1975, pp. 305-387. 
[7] G. Dore \& A. Venni, "On the closedness of the sum of two closed operators", Math. Z., 196, 1987, pp. 189-201.

[8] A. Favini, R. Labbas, S. Maingot, K. Lemrabet \& H. Sidibé, "Resolution and Optimal Regularity for a Biharmonic Equation with Impedance Boundary Conditions and Some Generalizations", Discrete and Continuous Dynamical Systems, Vol. 33, 11-12, 2013, pp. 4991-5014.

[9] A. Favini, R. Labbas, S. Maingot, H. Tanabe \& A. Yagi, "A simplified approach in the study of elliptic differential equations in UMD spaces and new applications", Funkc. Ekv., 451, 2008, pp. 165-187.

[10] A. Favini, R. Labbas, S. Maingot, H. Tanabe \& A. Yagi, "Complete abstract differential equations of elliptic type in UMD spaces", Funkc. Ekv., 49, 2006, pp. 193-214.

[11] G. Geymonat \& F. Krasucki, "Analyse asymptotique du comportement en flexion de deux plaques collées", C. R. Acad. Sci. Paris - serie II b, Volume 325, Issue 6, , 1997, pp. 307-314.

[12] D. Gilbarg \& N. Trudinger, Elliptic partial differential equations of second order, Classics in Mathematics, Springer-Verlag, Berlin, 2001.

[13] P. Grisvard, "Spazi di tracce e applicazioni", Rendiconti di Matematica, (4) Vol.5, Serie VI, 1972, pp. 657-729.

[14] Z. GuO, B. LAI \& D. YE, "Revisiting the biharmonic equation modelling electrostatic actuation in lower dimensions", Proc. Amer. Math. Soc., 142, 2014, pp. 2027-2034.

[15] M. HAase, The functional calculus for sectorial Operators, Birkhauser, 2006.

[16] M. HAASE, "Functional calculus for groups and applications to evolution equations", Journal of Evolution Equations, Volume 7, Issue 3, 2007, pp. 529-554.

[17] M. A. Jaswon \& G. T. Symm, "Integral equation methods in potential theory and elastostatics", Academic Press, New York, San Francisco, London, 1977, pp. 1-10.

[18] P. C. Kunstmann \& L. Weis, "New criteria for the $H^{\infty}$-calculus and the Stokes operator on bounded Lipschitz domains", Journal of Evolution Equations, Volume 17, Issue 1, 2017, pp. 387-409.

[19] R. Labbas, K. Lemrabet, S. Maingot \& A. Thorel, "Generalized linear models for population dynamics in two juxtaposed habitats", Discrete and Continuous Dynamical Systems A, Volume 39, Number 5, 2019, pp. 2933-2960.

[20] R. Labbas, S. Maingot, D. Manceau \& A. Thorel, "On the regularity of a generalized diffusion problem arising in population dynamics set in a cylindrical domain", Journal of Mathematical Analysis and Applications, 450, 2017, pp. 351-376.

[21] C. Le Merdy, "A sharp equivalence between $H^{\infty}$ functional calculus and square function estimates", Journal of Evolution Equations, Volume 12, Issue 4, 2012, pp. 789-800.

[22] K. Limam, R. Labbas, K. Lemrabet, A. Medeghri \& M. Meisner, "On Some Transmission Problems Set in a Biological Cell, Analysis and Resolution", Journal of Differential Equations, Volume 259, issue 7, 2015, p. 2695-2731.

[23] F. LIN \& Y. YANG, "Nonlinear non-local elliptic equation modelling electrostatic actuation", Proceedings of the Royal Society of London A, 463, 2007, pp. 1323-1337.

[24] J.-L. Lions \& J. Peetre, "Sur une classe d'espaces d'interpolation", Publications mathématiques de l'I.H.É.S., tome 19, 1964, pp. 5-68. 
[25] A. LunARDi, Analytic semigroups and optimal regularity in parabolic problems, Birkhauser, Basel, Boston, Berlin, 1995.

[26] F.L. OCHOA, "A generalized reaction-diffusion model for spatial structures formed by motile cells", BioSystems, 17, 1984, pp. 35-50.

[27] A. PAZY, Semigroups of linear operators and applications to partial differential equations, Springer-Verlag, New York, 1983.

[28] J. Prüss \& H. Sohr, "On operators with bounded imaginary powers in Banach spaces", Mathematische Zeitschrift, Springer-Verlag, Math. Z., 203, 1990, pp. 429-452.

[29] J. PRÜss \& H. SoHR, "Imaginary powers of elliptic second order differential operators in $L^{p}$-spaces", Hiroshima Math. J., 23, no. 1, 1993, pp. 161-192.

[30] J.L. Rubio De Francia, Martingale and integral transforms of Banach space valued functions, in: J. Bastero, M. San Miguel (Eds.), Probability and Banach Spaces, Zaragoza, 1985, in: Lecture Notes in Math., vol. 1221, Springer-Verlag, Berlin, 1986, pp. 195-222.

[31] H. Saker \& N. Bouselsal, "On the bilaplacian problem with nonlinear boundary conditions", Indian J. Pure Appl. Math., Volume 47, Issue 3, 2016, pp. 425-435.

[32] I. Titeux \& E. Sanchez-Palencia, "Conditions de transmission pour les jonctions de plaques minces", C. R. Acad. Sci. Paris - serie II b, Volume 325, Issue 10, 1997, pp. 563-570.

[33] H. TRIEBEL, Interpolation theory, function Spaces, differential Operators, North-Holland publishing company Amsterdam New York Oxford, 1978. 